\title{
Data report: grain size distribution of the late Cenozoic hemipelagic mud from Site $\mathrm{C} 0011^{1}$
}

\author{
Yu Saitoh ${ }^{2}$
}

\section{Chapter contents}

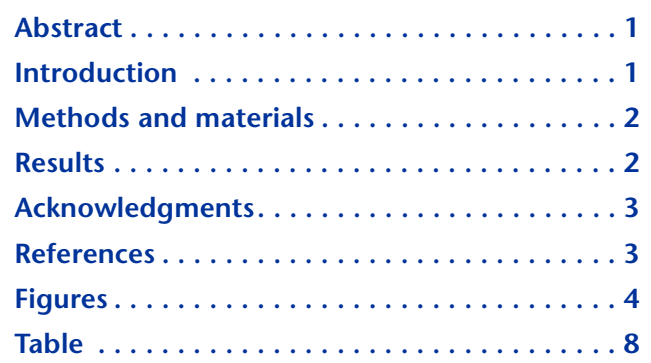

${ }^{1}$ Saitoh, Y., 2014. Data report: grain size distribution of the late Cenozoic hemipelagic mud from Site C0011. In Henry, P., Kanamatsu, T., Moe, K., and the Expedition 333 Scientists, Proc. IODP, 333: Tokyo (Integrated Ocean Drilling Program Management International, Inc.). doi:10.2204/iodp.proc.333.203.2014

${ }^{2}$ Center for Advanced Marine Core Research, Kochi University, B200 Monobe, Nankoku City, Kochi 783-8502, Japan. jm-yu-saitoh@kochi-u.ac.jp

\begin{abstract}
We performed grain size analyses of late Cenozoic hemipelagic mud samples from Holes C0011C and C0011D recovered during Integrated Ocean Drilling Program Expedition 333 (December 2010-January 2011). Hemipelagic mud grains are coarsest in the uppermost $94 \mathrm{~m}$ interval; the median diameter is $\sim 7.2 \phi$, although frequency distributions in the interval from 42 to 94 meters below seafloor (mbsf) are exceptionally broadened and flattened relative to the other horizons. Grain size fines downhole with fluctuations in the interval between 94 and 165 mbsf, where median grain size diameter fines downcore from 7 to $8 \phi$. Hemipelagic mud grains are finest in the interval from 165 to 380 mbsf. In this interval, median grain size diameter is consistently $\sim 8 \phi$, although coarsening excursions are present between 214 and 254 mbsf and between 351 and 363 mbsf.
\end{abstract}

\section{Introduction}

The purpose of this study is to provide information about grain sizes of hemipelagic mud in Holes C0011C and C0011D. Grain size distribution is one of the most important properties of sediment and is fundamental data used in studies of many fields.

Grain size distribution provides information about the origin and formative processes of sediment, including provenance, depositional environment, type and intensity of transport mechanisms, and depositional processes (e.g., Visher, 1969; McLaren, 1981; MacLaren and Bowles, 1985; Rea and Hovan, 1995; Holz et al., 2004). Therefore, grain size distribution can be a useful proxy in paleoenvironmental studies, especially when combined with other proxies.

Furthermore, physical properties of mud or mudstone are strongly influenced by grain size (Aplin and Macquaker, 2011). Hence, grain size distribution of the sediment deposited on the Philippine Sea plate at the Nankai Trough in Holes C0011C and C0011D will be an important data set when interpreting the downhole variation of several physical properties and the subsequent evolution of these properties during subduction (see the "Site C0011" chapter [Expedition 333 Scientists, 2012]). 


\section{Methods and materials}

\section{Materials}

During Expedition 333, an ocean-floor sediment succession, mostly hemipelagic mud with intercalated ash layers, was sampled from the surface to 379.93 meters below seafloor (mbsf). A total of 277 samples $(\sim 10 \mathrm{~mL}$, covering a depth interval of 2 $\mathrm{cm}$ ) were collected from almost every $150 \mathrm{~cm}$ core section. Sand and volcanic ash layers were avoided during sampling; however, a few samples accidentally contain a significant amount of ash. A total of 276 samples were analyzed in this study. The average depth interval between samples is $1.38 \mathrm{~m}$.

\section{Sample preparation}

About $50 \mathrm{mg}$ of each sediment sample, which was not dried or crushed, was soaked with $12 \mathrm{~mL}$ of 5.5 $\mathrm{g} / \mathrm{L}$ sodium hexametaphosphate dispersant for $>24 \mathrm{~h}$ and then was sonicated and well dispersed by hand shaking. Stiff lumps were gently crushed with a thin wood stick within the dispersant. To check for errors originating from nonhomogeneity of the samples, three aliquots using a different part of each sample block were made for 27 samples, selected from each consecutive 10 samples (Table T1).

\section{Grain size analysis}

Grain size analysis was carried out using a Malvern Mastersizer 2000 laser diffractometer with an automated sample dispersion unit (Hydro 2000S) installed at the Kochi Core Center (Japan). Principles of the instrument are described in Sperazza et al. (2004). We adopted the procedure and analytical setting proposed by Sperazza et al. (2004) with some modification. In this study, samples dispersed in advance were poured into the dispersion unit, with pump speed and intensity of sonication set to 2000 rpm and 20\%, respectively. The input amount of the samples was controlled so that obscuration fell within the $15 \%-20 \%$ range. The grain reflectance index and dispersant were set to 1.555 and 1.33, respectively. Particle absorption was set to 1 . Each measurement run was set for $12 \mathrm{~s}$, or 12,000 snaps, and repeated five times. Grain size analyses reported in this paper are the fifth result of the repetition series. Prior to accepting an analysis, we visually inspected the output from each of the five runs for consistency.

Results were compiled with Malvern's Mastersizer 2000 software as the volume frequency of 100 size classes. The boundaries between classes are set as a geometric series from 0.02 to $2000 \mu \mathrm{m}$. For convenience, we used the phi $(\phi)$ scale in the following text, table, and figures. The grain size unit $\phi$ is derived as follows:

$$
\phi=-\log _{2}\left(d / d_{0}\right),
$$

where $d$ (in millimeters) is the diameter of a particle and $d_{0}$ is a reference diameter equal to $1 \mathrm{~mm}$.

\section{Results}

Results are shown in Figures F1 and F2 and in Table T1. Raw data of frequency distribution is provided in GRAINSIZ in "Supplementary material."

Errors derived from nonhomogeneity of samples were mostly $<0.2 \phi$, except for mode diameter, in terms of standard deviation of each representative value derived from measurements of three different aliquots from each of 27 selected samples (Fig. F3). Correlation of standard deviation to 10-percentile and median diameter is a nonzero value by $>0.99$ probability, and that to mean diameter is $>0.98$ probability (Fig. F4). Smaller diameters tend to show smaller standard deviation values. Thus, a difference of $\sim 0.2 \phi$ in median diameter between different samples can be regarded significant if the median diameter is $<7 \phi$.

Hemipelagic mud grains are coarsest in the uppermost $94 \mathrm{~m}$ interval, with median diameters of $\sim 7.2 \phi$. However, the 42-94 mbsf interval is characterized by a broadened grain size frequency distribution. In contrast, median and 10-percentile diameters differ little from the uppermost $42 \mathrm{~m}$ interval; 90percentile diameter is obviously coarser between 42 and 94 mbsf than in the uppermost interval. Frequency distributions are also flattened because of the low frequency of modal grain size (Fig. F1).

Grain size generally fines downcore with fluctuations in the 94 to 165 mbsf interval. Median and 90-percentile diameters fine from 7 to $8 \phi$ and from 5 to $6 \phi$, respectively, whereas 10-percentile diameter fines slightly from 9.6 to $10 \phi$ (Fig. F2A).

Hemipelagic mud grains are finest from 165 to 380 mbsf (Figs. F1, F2). In this interval, grain size shows narrow, sharp frequency distribution, and median diameter is almost steady at $\sim 8 \phi$. A few excursions were observed between 254 and 214 mbsf, where median diameter coarsens to $6.5 \phi$, and between 351 and 363 mbsf, where median diameter coarsens to $5.3 \phi$. 


\section{Acknowledgments}

This research used samples and data provided by the Integrated Ocean Drilling Program (IODP). Funding was provided by Japan Drilling Earth Science Consortium and Center for Deep Earth Exploration.

\section{References}

Aplin, A.C., and Macquaker, J.H.S., 2011. Mudstone diversity: origin and implications for source, seal, and reservoir properties in petroleum systems. AAPG Bull., 95(12):2031-2059. doi:10.1306/03281110162

Expedition 333 Scientists, 2012. Site C0011. In Henry, P., Kanamatsu, T., Moe, K., and the Expedition 333 Scientists, Proc. IODP, 333: Tokyo (Integrated Ocean Drilling Program Management International, Inc.). doi:10.2204/iodp.proc.333.104.2012

Holz, C., Stuut, J.-B.W., and Henrich, R., 2004. Terrigenous sedimentation processes along the continental margin off NW Africa: implications from grain-size analysis of seabed sediments. Sedimentology, 51(5):1145-1154. doi:10.1111/j.1365-3091.2004.00665.x

McLaren, P., 1981. An interpretation of trends in grain size measures. J. Sediment. Res., 51(2):611-624. doi:10.1306/ 212F7CF2-2B24-11D7-8648000102C1865D
McLaren, P., and Bowles, D., 1985. The effects of sediment transport on grain-size distributions. J. Sediment. Res., 55(4):457-470. doi:10.1306/212F86FC-2B24-11D78648000102C1865D

Rea, D.K., and Hovan, S.A., 1995. Grain size distribution and depositional processes of the mineral component of abyssal sediments: lessons from the North Pacific. Paleoceanography, 10(2):251-258. doi:10.1029/ 94PA03355

Sperazza, M., Moore, J.N., and Hendrix, M.S., 2004. Highresolution particle size analysis of naturally occurring very fine-grained sediment through laser diffractometry. J. Sediment. Res., 74(5):736-743. doi:10.1306/ 031104740736

Visher, G.S., 1969. Grain size distributions and depositional processes. J. Sediment. Res., 39(3):1074-1106. doi:10.1306/74D71D9D-2B21-11D78648000102C1865D

Initial receipt: 4 July 2013

Acceptance: 15 January 2014

Publication: 24 April 2014

MS 333-203 
Figure F1. Downcore variation of frequency distribution of grain size, Site C0011.

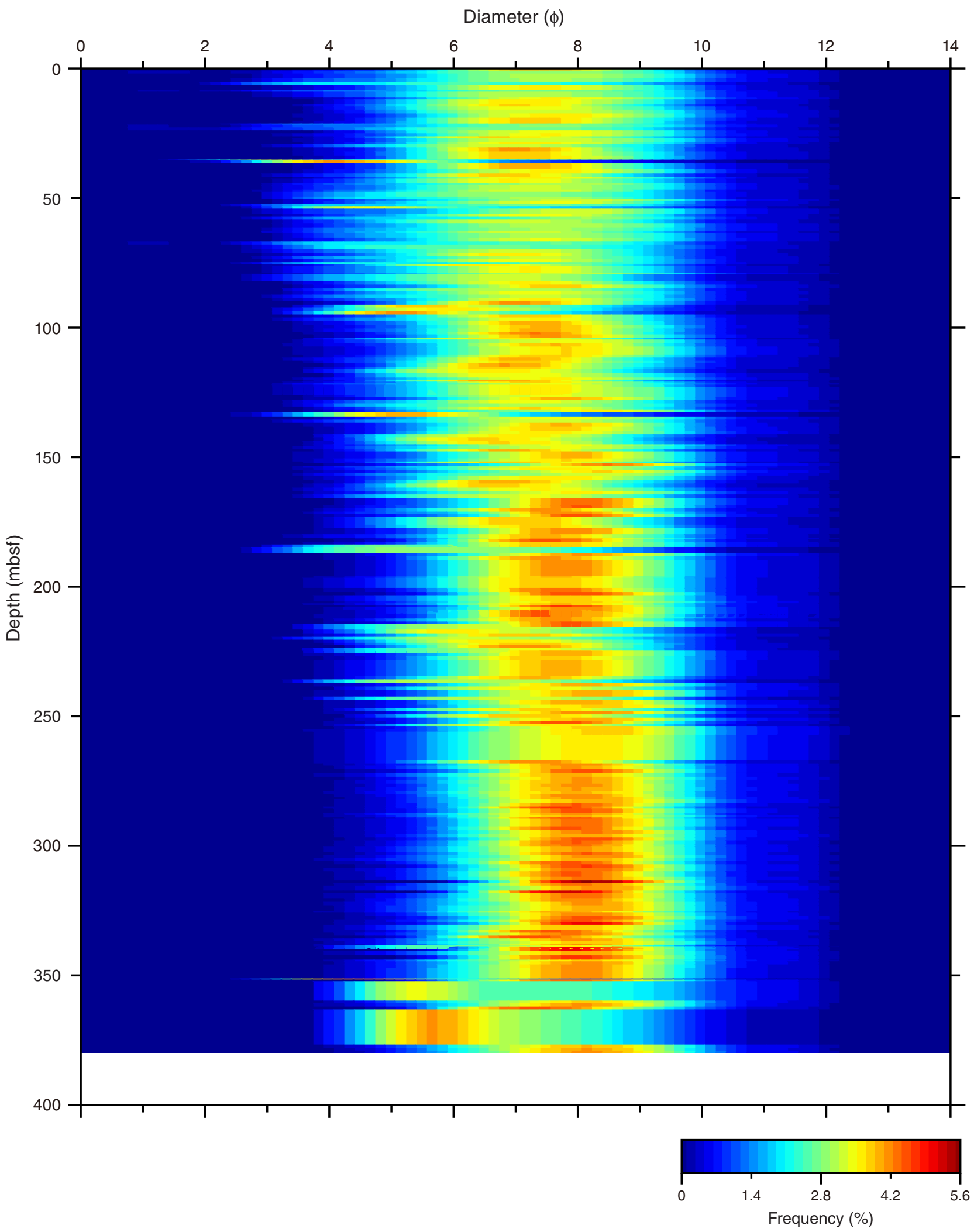


Figure F2. Downcore variation of representative values of grain size distributions, Site C0011. A. 10-, 50(median), and 90-percentile diameter. B. Mode and mean diameter.
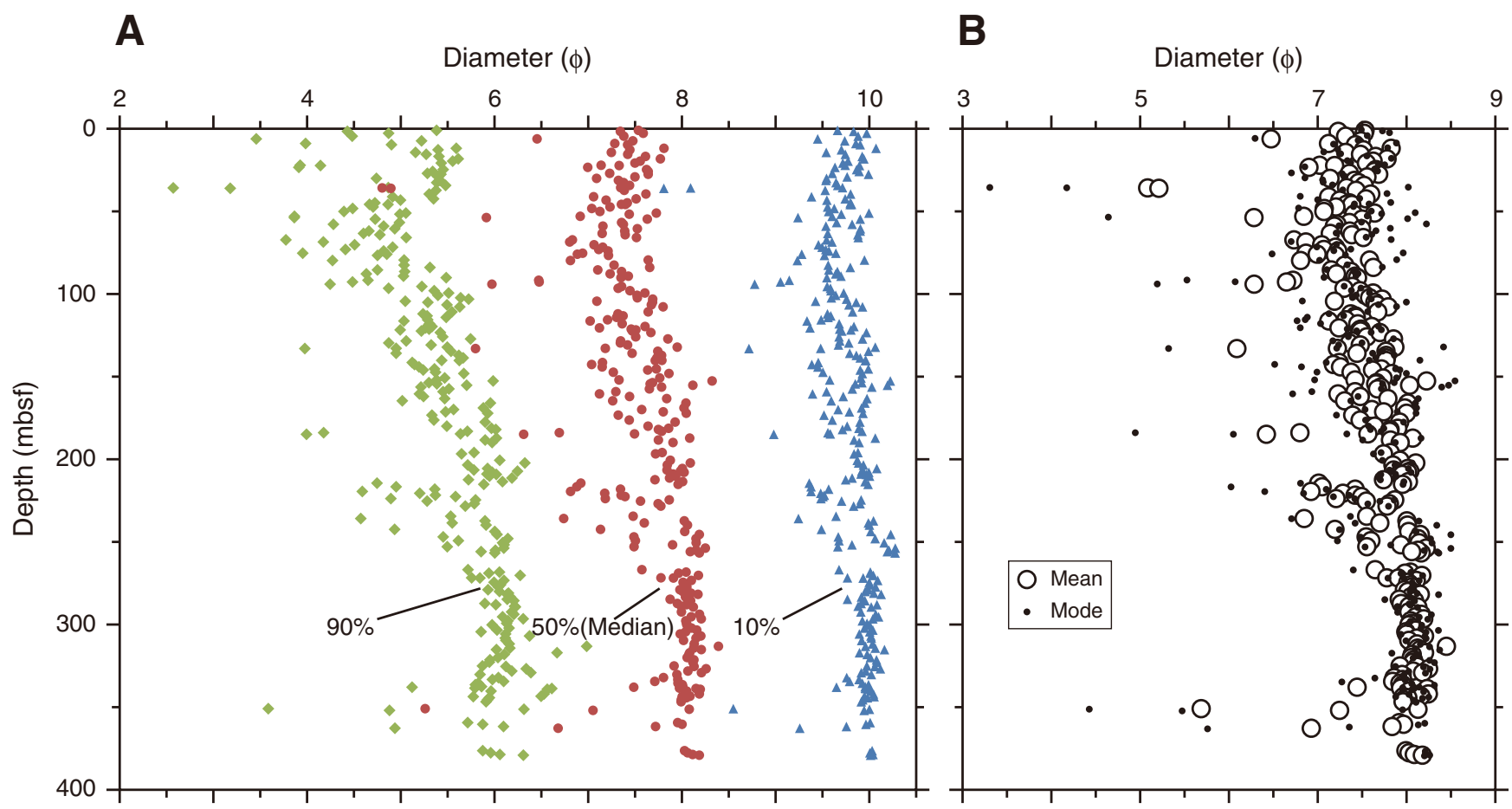
Figure F3. Histgram of standard deviation (S.D.) of representative values derived from measured values of three different aliquots from 27 selected samples, Site C0011. A. 10-percentile diameter. B. 50-percentile (median) diameter. C. 90-percentile diameter. D. Modal diameter. E. Mean diameter.

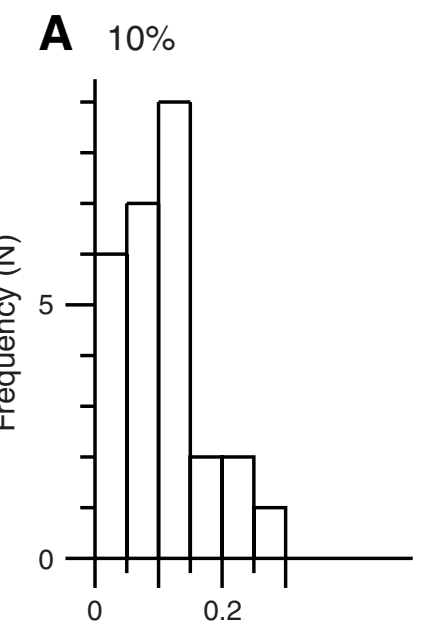

S.D. $(\phi)$
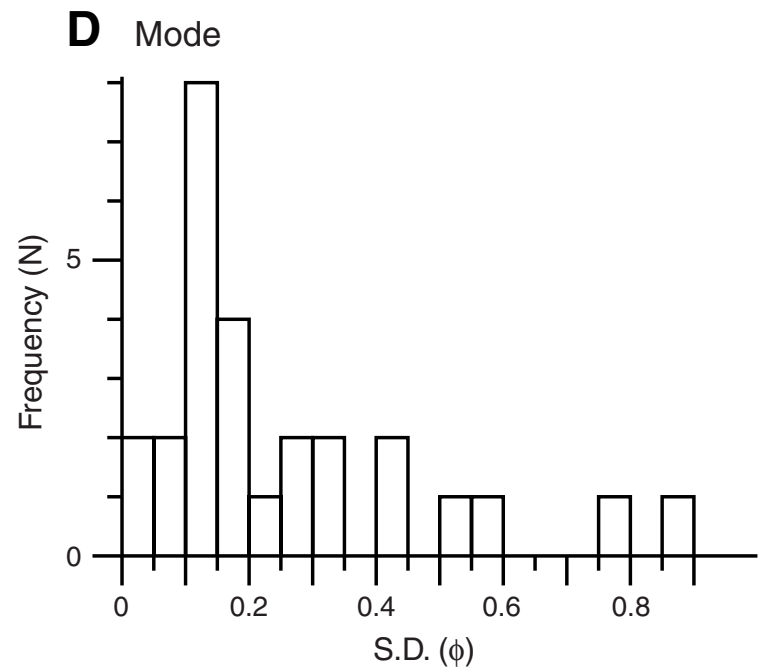

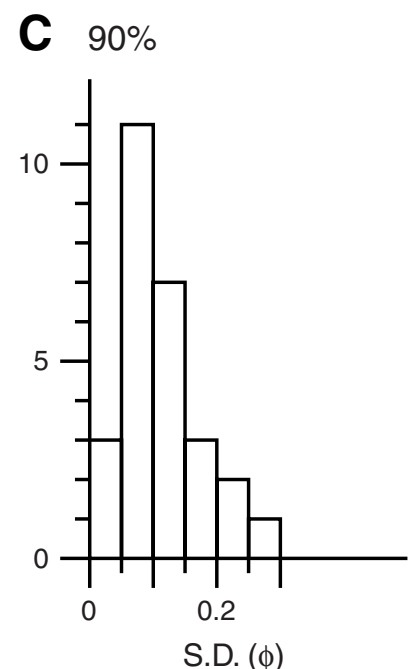

E Mean

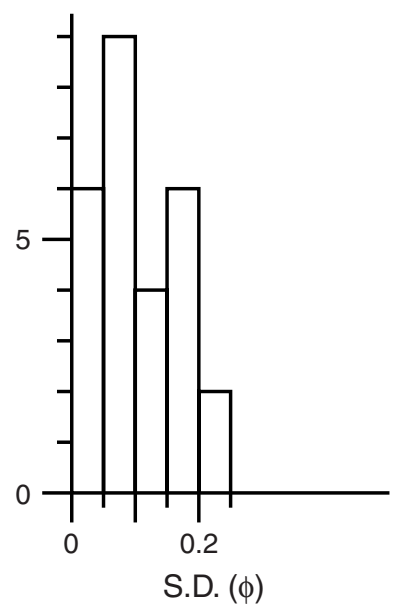


Figure F4. Correlation between average of each representative value and its standard deviation (S.D.) derived from measured values of three different aliquots from 27 selecteds samples, Site C0011. A. 10-percentile diameter. B. 50-percentile (median) diameter. C. 90-percentile diameter. D. Modal diameter. E. Mean diameter.
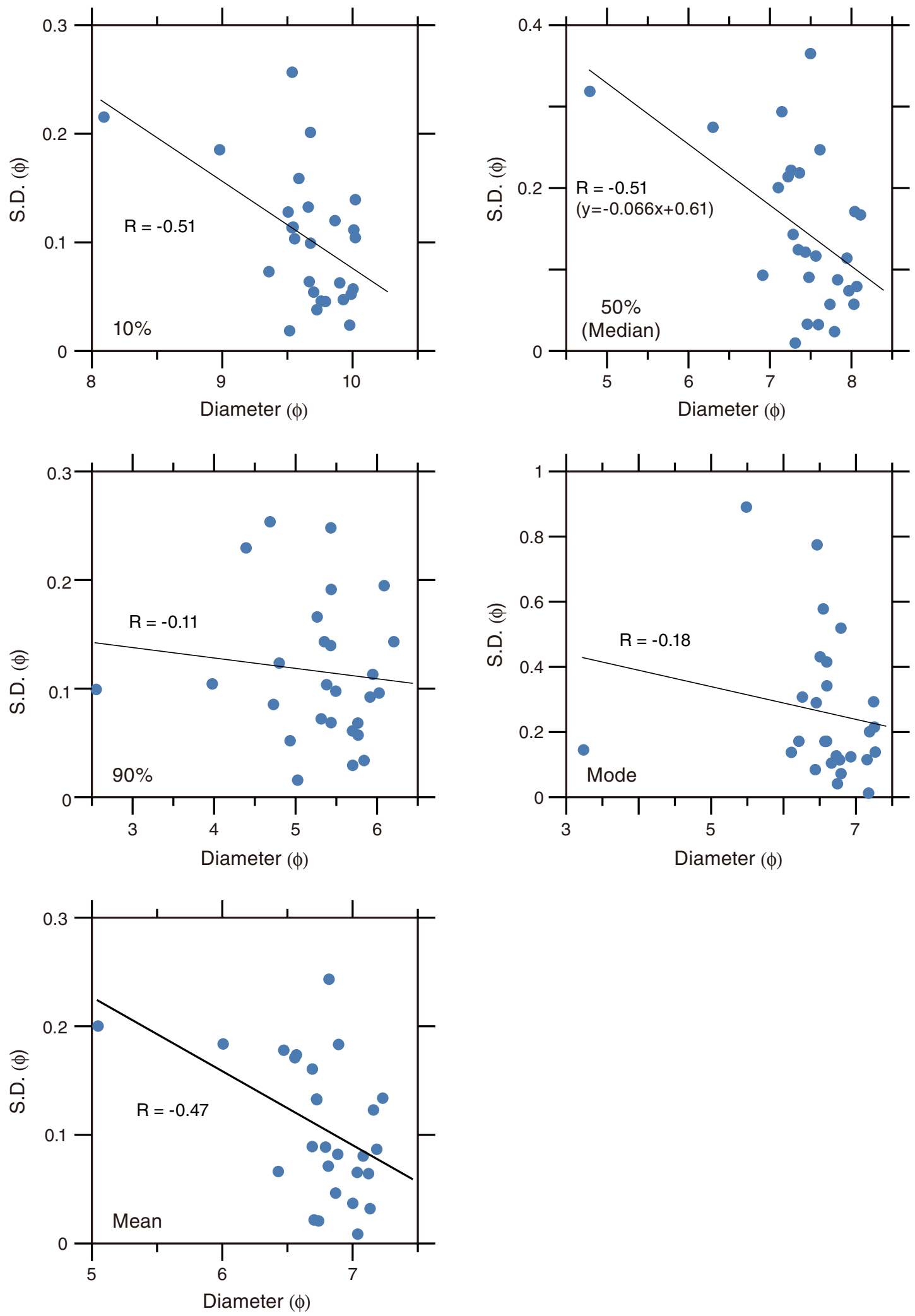
Table T1. Representative values of grain size distribution, Site C0011. (Continued on next five pages.)

\begin{tabular}{|c|c|c|c|c|c|c|c|c|}
\hline \multirow[b]{2}{*}{ Sample } & \multirow[b]{2}{*}{ Hole, core, section, interval $(\mathrm{cm})$} & \multicolumn{2}{|c|}{ Core depth CSF-B (m) } & \multirow[b]{2}{*}{ 10-percentile } & \multirow[b]{2}{*}{ 50-percentile } & \multirow[b]{2}{*}{ 90-percentile } & \multirow[b]{2}{*}{ Mean } & \multirow[b]{2}{*}{ Mode } \\
\hline & & Top & Bottom & & & & & \\
\hline & $333-$ & & & & & & & \\
\hline 1 & C0011C-1H-1W, 44-46 & 0.422 & 0.441 & 9.66 & 7.53 & 5.37 & 7.53 & 7.49 \\
\hline 2 & C0011C-1H-3W, 24-26 & 1.040 & 1.060 & 9.84 & 7.34 & 4.41 & 7.23 & 7.73 \\
\hline 3 & C0011C-1H-5W, 20-22 & 2.181 & 2.201 & 9.98 & 7.58 & 4.86 & 7.52 & 7.81 \\
\hline 4 & C0011C-2H-1W, 49-51 & 3.971 & 3.990 & 9.88 & 7.37 & 4.47 & 7.31 & 7.56 \\
\hline 5 & C0011C-2H-3W, 81-83 & 5.613 & 5.633 & 9.45 & 6.45 & 3.44 & 6.46 & 6.29 \\
\hline 6 & $\mathrm{C} 0011 \mathrm{C}-2 \mathrm{H}-4 \mathrm{~W}, 60-62$ & 6.775 & 6.795 & 9.74 & 7.47 & 5.21 & 7.48 & 7.46 \\
\hline 7 & C0011C-2H-6W, 88-90 & 8.413 & 8.432 & 9.86 & 7.28 & 3.97 & 7.12 & 7.89 \\
\hline 8 & C0011C-2H-7W, 22-24 & 9.134 & 9.153 & 9.91 & 7.41 & 4.89 & 7.42 & 7.18 \\
\hline 9 & C0011C-2H-9W, 109-111 & 11.324 & 11.343 & 10.08 & 7.80 & 5.58 & 7.83 & 7.79 \\
\hline 10 & C0011C-2H-11W, 62-64 & 12.236 & 12.256 & 9.64 & 7.32 & 5.27 & 7.41 & 6.96 \\
\hline 10 rep. & & & & 9.73 & 7.46 & 5.43 & 7.54 & 7.19 \\
\hline 10 rep. & & & & 9.74 & 7.53 & 5.45 & 7.58 & 7.53 \\
\hline 10 average & & & & 9.70 & 7.44 & 5.39 & 7.51 & 7.22 \\
\hline 10 S.D. & & & & 0.05 & 0.11 & 0.10 & 0.09 & 0.29 \\
\hline 11 & C0011C-3H-1W, 86-88 & 13.808 & 13.827 & 9.54 & 7.24 & 5.14 & 7.31 & 7.07 \\
\hline 12 & C0011C-3H-2W, 67-69 & 14.955 & 14.973 & 9.75 & 7.42 & 5.26 & 7.48 & 7.26 \\
\hline 13 & C0011C-3H-3W, 62-64 & 16.232 & 16.251 & 9.93 & 7.61 & 5.39 & 7.65 & 7.55 \\
\hline 14 & C0011C-3H-6W, 74-76 & 17.712 & 17.731 & 9.94 & 7.77 & 5.60 & 7.78 & 7.83 \\
\hline 15 & C0011C-3H-7W, 82-84 & 19.108 & 19.127 & 9.78 & 7.55 & 5.54 & 7.62 & 7.33 \\
\hline 16 & C0011C-3H-9W, 65-67 & 20.287 & 20.306 & 9.72 & 7.49 & 5.42 & 7.55 & 7.37 \\
\hline 17 & C0011D-1H-1W, 65-67 & 21.611 & 21.630 & 9.74 & 7.13 & 3.91 & 7.02 & 7.76 \\
\hline 18 & C0011C-3H-10W, 81-83 & 21.762 & 21.781 & 9.90 & 7.32 & 4.13 & 7.19 & 7.69 \\
\hline 19 & C0011D-1H-2W, 57-59 & 22.862 & 22.881 & 9.63 & 6.99 & 3.89 & 6.89 & 6.85 \\
\hline 20 & C0011D-1H-3W, 57-59 & 24.189 & 24.207 & 9.94 & 7.73 & 5.54 & 7.75 & 7.94 \\
\hline 20 rep. & & & & 9.94 & 7.76 & 5.56 & 7.77 & 7.94 \\
\hline 20 rep. & & & & 9.73 & 7.37 & 5.22 & 7.44 & 7.04 \\
\hline 20 average & & & & 9.87 & 7.62 & 5.44 & 7.65 & 7.64 \\
\hline 20 S.D. & & & & 0.12 & 0.22 & 0.19 & 0.18 & 0.52 \\
\hline 21 & C0011D-1H-5W, 18-20 & 25.355 & 25.374 & 9.89 & 7.63 & 5.33 & 7.63 & 7.68 \\
\hline 22 & C0011D-1H-6W, 2-4 & 26.526 & 26.545 & 9.61 & 7.22 & 5.27 & 7.35 & 6.70 \\
\hline 23 & C0011D-1H-7W, 38-40 & 27.072 & 27.09 & 10.00 & 7.63 & 5.42 & 7.68 & 7.28 \\
\hline 24 & C0011D-1H-8W, 87-89 & 28.656 & 28.675 & 9.77 & 7.49 & 5.39 & 7.55 & 7.31 \\
\hline 25 & C0011D-1H-9W, 40-42 & 29.550 & 29.569 & 9.55 & 7.08 & 4.72 & 7.13 & 6.85 \\
\hline 26 & C0011D-2H-1W, 28-30 & 30.772 & 30.792 & 9.54 & 7.32 & 5.41 & 7.41 & 7.05 \\
\hline 27 & C0011D-2H-2W, 31-33 & 32.158 & 32.177 & 9.65 & 7.37 & 5.37 & 7.46 & 7.06 \\
\hline 28 & C0011D-2H-3W, 61-63 & 33.830 & 33.850 & 9.66 & 7.44 & 5.47 & 7.52 & 7.17 \\
\hline 29 & C0011D-2H-4W, 56-58 & 35.162 & 35.182 & 9.72 & 7.34 & 4.85 & 7.32 & 8.02 \\
\hline 30 & C0011D-2H-4W, 76-78 & 35.357 & 35.376 & 7.86 & 4.47 & 2.44 & 4.84 & 3.13 \\
\hline 30 rep. & & & & 8.11 & 4.88 & 2.61 & 5.12 & 3.41 \\
\hline 30 rep. & & & & 8.29 & 5.01 & 2.61 & 5.22 & 3.32 \\
\hline 30 average & & & & 8.09 & 4.79 & 2.55 & 5.06 & 3.29 \\
\hline 30 S.D. & & & & 0.22 & 0.28 & 0.10 & 0.20 & 0.14 \\
\hline 31 & C0011D-2H-4W, 98-100 & 35.571 & 35.590 & 7.80 & 4.88 & 3.16 & 5.19 & 4.16 \\
\hline 32 & C0011D-2H-7W, 59-61 & 36.806 & 36.825 & 9.53 & 7.38 & 5.36 & 7.43 & 7.28 \\
\hline 33 & C0011D-2H-9W, 39-41 & 39.052 & 39.071 & 9.80 & 7.61 & 5.29 & 7.59 & 7.78 \\
\hline 34 & C0011D-3H-1W, 74-76 & 40.702 & 40.722 & 9.38 & 7.05 & 4.90 & 7.12 & 6.80 \\
\hline 35 & C0011D-3H-2W, 70-72 & 42.008 & 42.027 & 9.74 & 7.51 & 5.34 & 7.54 & 7.47 \\
\hline 36 & C0011D-3H-3W, 10-12 & 42.777 & 42.796 & 9.55 & 7.18 & 4.98 & 7.24 & 7.00 \\
\hline 37 & C0011D-3H-4W, 41-43 & 44.419 & 44.438 & 9.80 & 7.41 & 4.71 & 7.35 & 7.82 \\
\hline 38 & C0011D-3H-6W, 33-35 & 45.340 & 45.359 & 9.81 & 7.35 & 4.65 & 7.32 & 7.58 \\
\hline 39 & C0011D-3H-6W, 36-38 & 45.369 & 45.388 & 9.80 & 7.39 & 4.85 & 7.38 & 7.41 \\
\hline 40 & C0011D-3H-7W, 55-57 & 46.897 & 46.916 & 9.67 & 7.43 & 4.95 & 7.39 & 7.88 \\
\hline 40 rep. & & & & 9.48 & 7.07 & 4.45 & 7.05 & 7.10 \\
\hline 40 rep. & & & & 9.52 & 7.17 & 4.67 & 7.16 & 7.23 \\
\hline 40 average & & & & 9.56 & 7.23 & 4.69 & 7.20 & 7.40 \\
\hline 40 S.D. & & & & 0.10 & 0.19 & 0.25 & 0.17 & 0.41 \\
\hline 41 & C0011D-3H-8W, 9-11 & 47.813 & 47.832 & 9.63 & 7.03 & 4.47 & 7.07 & 6.78 \\
\hline 42 & C0011D-4H-1W, 82-84 & 49.653 & 49.669 & 9.57 & 7.12 & 4.38 & 7.06 & 7.60 \\
\hline 43 & C0011D-4H-2W, 67-69 & 50.661 & 50.677 & 10.00 & 7.72 & 5.04 & 7.64 & 8.10 \\
\hline 44 & C0011D-4H-3W, 8-10 & 51.322 & 51.338 & 9.70 & 7.43 & 4.98 & 7.40 & 7.72 \\
\hline 45 & C0011D-4H-6W, 59.5-61.5 & 52.592 & 52.609 & 9.60 & 6.91 & 3.85 & 6.83 & 7.56 \\
\hline 46 & C0011D-4H-7W, 34.5-36.5 & 53.345 & 53.361 & 9.24 & 5.90 & 3.85 & 6.27 & 4.63 \\
\hline 47 & C0011D-4H-8W, 8-10 & 54.261 & 54.277 & 9.91 & 7.63 & 4.71 & 7.50 & 8.02 \\
\hline 48 & C0011D-5H-1W, 88-90 & 56.341 & 56.360 & 9.65 & 7.35 & 4.97 & 7.35 & 7.49 \\
\hline 49 & C0011D-5H-2W, 66-68 & 57.474 & 57.493 & 9.82 & 7.37 & 4.26 & 7.21 & 8.23 \\
\hline 50 & C0011D-5H-3W, 38-40 & 58.554 & 58.573 & 9.49 & 7.11 & 4.72 & 7.13 & 7.20 \\
\hline 50 rep. & & & & 9.31 & 6.91 & 4.94 & 7.04 & 6.49 \\
\hline
\end{tabular}


Table T1 (continued). (Continued on next page.)

\begin{tabular}{|c|c|c|c|c|c|c|c|c|}
\hline \multirow[b]{2}{*}{ Sample } & \multirow[b]{2}{*}{ Hole, core, section, interval $(\mathrm{cm})$} & \multicolumn{2}{|c|}{ Core depth CSF-B (m) } & \multirow[b]{2}{*}{ 10-percentile } & \multirow[b]{2}{*}{ 50-percentile } & \multirow[b]{2}{*}{ 90-percentile } & \multirow[b]{2}{*}{ Mean } & \multirow[b]{2}{*}{ Mode } \\
\hline & & Top & Bottom & & & & & \\
\hline 50 rep. & & & & 9.82 & 7.42 & 4.75 & 7.37 & 8.04 \\
\hline 50 average & & & & 9.54 & 7.15 & 4.80 & 7.18 & 7.24 \\
\hline 50 S.D. & & & & 0.26 & 0.26 & 0.12 & 0.17 & 0.77 \\
\hline 51 & C0011D-5H-4W, 60-62 & 60.107 & 60.126 & 9.91 & 7.52 & 4.92 & 7.49 & 7.82 \\
\hline 52 & C0011D-5H-6W, 68-70 & 61.555 & 61.574 & 9.91 & 7.38 & 4.65 & 7.36 & 7.66 \\
\hline 53 & C0011D-5H-7W, 76-78 & 62.983 & 63.002 & 9.56 & 7.14 & 4.59 & 7.14 & 7.21 \\
\hline 54 & C0011D-5H-8W, 18-20 & 63.772 & 63.791 & 9.88 & 7.39 & 4.76 & 7.38 & 7.61 \\
\hline 55 & C0011D-6H-1W, 48-50 & 65.456 & 65.475 & 9.89 & 7.51 & 5.04 & 7.51 & 7.55 \\
\hline 56 & C0011D-6H-2W, 59-61 & 66.905 & 66.924 & 9.54 & 6.82 & 3.76 & 6.72 & 7.83 \\
\hline 57 & C0011D-6H-3W, 50-52 & 68.154 & 68.173 & 9.60 & 6.80 & 4.16 & 6.86 & 6.70 \\
\hline 58 & C0011D-6H-5W, 90-92 & 69.878 & 69.897 & 9.46 & 7.06 & 4.49 & 7.04 & 7.21 \\
\hline 59 & C0011D-6H-6W, 105-107 & 71.360 & 71.379 & 9.50 & 7.15 & 4.90 & 7.19 & 6.99 \\
\hline 60 & C0011D-6H-7W, 105-107 & 72.704 & 72.724 & 9.41 & 6.92 & 4.16 & 6.87 & 7.19 \\
\hline 60 rep. & & & & 9.64 & 7.26 & 4.62 & 7.23 & 7.40 \\
\hline 60 rep. & & & & 9.56 & 7.13 & 4.41 & 7.09 & 7.53 \\
\hline 60 average & & & & 9.54 & 7.11 & 4.39 & 7.06 & 7.37 \\
\hline 60 S.D. & & & & 0.11 & 0.18 & 0.23 & 0.18 & 0.17 \\
\hline 61 & C0011D-6H-8W, 62-63 & 73.645 & 73.655 & 9.57 & 7.21 & 4.80 & 7.22 & 7.18 \\
\hline 62 & C0011D-7H-1W, 108-110 & 75.005 & 75.015 & 9.64 & 6.93 & 3.94 & 6.86 & 7.97 \\
\hline 63 & C0011D-7H-2W, 100-102 & 75.620 & 75.630 & 9.28 & 6.88 & 4.84 & 6.99 & 6.48 \\
\hline 64 & C0011D-7H-3W, 108-110 & 76.317 & 76.327 & 9.52 & 7.21 & 4.74 & 7.19 & 7.31 \\
\hline 65 & C0011D-8H-1W, 46-48 & 79.134 & 79.140 & 9.95 & 7.64 & 5.02 & 7.58 & 7.89 \\
\hline 66 & C0011D-8H-2W, 12-14 & 79.446 & 79.452 & 9.23 & 6.80 & 4.26 & 6.80 & 7.02 \\
\hline 67 & C0011D-9H-1W, 73-75 & 82.213 & 82.232 & 9.56 & 7.27 & 5.02 & 7.30 & 7.33 \\
\hline 68 & C0011D-9H-2W, 77-79 & 83.618 & 83.638 & 9.80 & 7.65 & 5.30 & 7.62 & 7.73 \\
\hline 69 & C0011D-9H-4W, 65-67 & 85.136 & 85.156 & 9.57 & 7.10 & 4.74 & 7.15 & 7.10 \\
\hline 70 & C0011D-9H-5W, 75-77 & 86.127 & 86.146 & 9.64 & 7.32 & 5.03 & 7.35 & 7.22 \\
\hline 70 rep. & & & & 9.80 & 7.47 & 5.05 & 7.47 & 7.80 \\
\hline 70 rep. & & & & 9.54 & 7.26 & 5.02 & 7.29 & 7.20 \\
\hline 70 average & & & & 9.66 & 7.35 & 5.03 & 7.37 & 7.40 \\
\hline 70 S.D. & & & & 0.13 & 0.11 & 0.02 & 0.09 & 0.34 \\
\hline 71 & C0011D-9H-6W, 74-76 & 87.493 & 87.513 & 9.64 & 7.23 & 4.62 & 7.21 & 7.45 \\
\hline 72 & C0011D-9H-7W, 79-81 & 88.904 & 88.924 & 9.70 & 7.42 & 5.01 & 7.42 & 7.43 \\
\hline 73 & C0011D-9H-8W, 54-56 & 89.851 & 89.870 & 9.55 & 7.34 & 5.47 & 7.44 & 7.07 \\
\hline 74 & C0011D-10H-1W, 96-98 & 91.411 & 91.430 & 9.15 & 6.46 & 4.63 & 6.71 & 5.52 \\
\hline 75 & C0011D-10H-2W, 68-70 & 92.455 & 92.474 & 9.05 & 6.47 & 4.47 & 6.64 & 6.06 \\
\hline 76 & C0011D-10H-3W, 70-72 & 93.817 & 93.836 & 8.78 & 5.96 & 4.23 & 6.27 & 5.18 \\
\hline 77 & C0011D-10H-4W, 73-75 & 95.169 & 95.188 & 9.55 & 7.35 & 5.21 & 7.39 & 7.30 \\
\hline 78 & C0011D-10H-6W, 51-53 & 96.308 & 96.327 & 9.59 & 7.31 & 4.86 & 7.29 & 7.49 \\
\hline 79 & C0011D-10H-7W, 63-65 & 97.756 & 97.774 & 9.59 & 7.44 & 5.34 & 7.47 & 7.38 \\
\hline 80 & C0011D-10H-8W, 68-70 & 99.141 & 99.160 & 9.74 & 7.59 & 5.43 & 7.60 & 7.62 \\
\hline 80 rep. & & & & 9.69 & 7.58 & 5.45 & 7.59 & 7.58 \\
\hline 80 rep. & & & & 9.76 & 7.63 & 5.61 & 7.67 & 7.54 \\
\hline 80 average & & & & 9.73 & 7.60 & 5.50 & 7.62 & 7.58 \\
\hline 80 S.D. & & & & 0.04 & 0.03 & 0.10 & 0.05 & 0.04 \\
\hline 81 & C0011D-11H-1W, 58-60 & 100.564 & 100.583 & 9.64 & 7.52 & 5.38 & 7.53 & 7.43 \\
\hline 82 & C0011D-11H-2W, 68-70 & 102.042 & 102.061 & 9.60 & 7.52 & 5.63 & 7.58 & 7.39 \\
\hline 83 & C0011D-11H-3W, 9-11 & 102.849 & 102.868 & 9.76 & 7.68 & 5.71 & 7.72 & 7.60 \\
\hline 84 & C0011D-11H-4W, 19-21 & 104.118 & 104.137 & 9.43 & 7.09 & 5.03 & 7.18 & 6.82 \\
\hline 85 & C0011D-11H-6W, 42-44 & 104.794 & 104.813 & 9.86 & 7.68 & 5.28 & 7.64 & 8.00 \\
\hline 86 & C0011D-11H-7W, 59-61 & 106.340 & 106.359 & 9.79 & 7.67 & 5.48 & 7.66 & 7.69 \\
\hline 87 & C0011D-11H-8W, 39-41 & 107.531 & 107.550 & 9.93 & 7.80 & 5.62 & 7.79 & 7.89 \\
\hline 88 & C0011D-12H-1W, 115-117 & 110.596 & 110.615 & 9.82 & 7.63 & 5.53 & 7.67 & 7.56 \\
\hline 89 & C0011D-12H-2W, 107-109 & 111.820 & 111.839 & 9.56 & 7.31 & 5.22 & 7.37 & 7.13 \\
\hline 90 & C0011D-12H-3W, 93-95 & 113.030 & 113.049 & 9.42 & 7.15 & 5.29 & 7.27 & 6.80 \\
\hline 90 rep. & & & & 9.73 & 7.45 & 5.09 & 7.45 & 7.56 \\
\hline 90 rep. & & & & 9.62 & 7.50 & 5.42 & 7.53 & 7.52 \\
\hline 90 average & & & & 9.59 & 7.37 & 5.27 & 7.42 & 7.29 \\
\hline 90 S.D. & & & & 0.16 & 0.19 & 0.17 & 0.13 & 0.43 \\
\hline 91 & C0011D-12H-4W, 46-48 & 113.921 & 113.940 & 9.56 & 7.30 & 5.47 & 7.43 & 6.88 \\
\hline 92 & C0011D-12H-6W, 101-103 & 115.427 & 115.446 & 9.49 & 7.20 & 5.27 & 7.31 & 6.86 \\
\hline 93 & C0011D-12H-7W, 33-35 & 116.127 & 116.146 & 9.34 & 7.01 & 5.02 & 7.12 & 6.76 \\
\hline 94 & C0011D-12H-8W, 64-66 & 117.814 & 117.833 & 9.68 & 7.36 & 5.29 & 7.45 & 7.03 \\
\hline 95 & C0011D-13H-1W, 82-84 & 119.488 & 119.499 & 9.84 & 7.60 & 5.32 & 7.61 & 7.68 \\
\hline 96 & C0011D-13H-2W, 80-82 & 120.323 & 120.335 & 9.37 & 7.11 & 5.25 & 7.23 & 6.80 \\
\hline 97 & C0011D-13H-3W, 63-65 & 121.063 & 121.075 & 9.68 & 7.45 & 5.29 & 7.49 & 7.33 \\
\hline 98 & C0011D-13H-4W, 46-48 & 121.563 & 121.575 & 9.81 & 7.50 & 4.98 & 7.47 & 7.72 \\
\hline 99 & C0011D-13H-6W, 63-65 & 122.235 & 122.247 & 9.71 & 7.47 & 5.21 & 7.48 & 7.44 \\
\hline 100 & C0011D-14H-1W, 85-87 & 125.740 & 125.758 & 9.51 & 7.26 & 5.29 & 7.35 & 6.99 \\
\hline
\end{tabular}


Table T1 (continued). (Continued on next page.)

\begin{tabular}{|c|c|c|c|c|c|c|c|c|}
\hline \multirow[b]{2}{*}{ Sample } & \multirow[b]{2}{*}{ Hole, core, section, interval $(\mathrm{cm})$} & \multicolumn{2}{|c|}{ Core depth CSF-B (m) } & \multirow[b]{2}{*}{ 10-percentile } & \multirow[b]{2}{*}{ 50-percentile } & \multirow[b]{2}{*}{ 90-percentile } & \multirow[b]{2}{*}{ Mean } & \multirow[b]{2}{*}{ Mode } \\
\hline & & Top & Bottom & & & & & \\
\hline 100 rep. & & & & 9.90 & 7.86 & 5.56 & 7.82 & 8.01 \\
\hline 100 rep. & & & & 9.63 & 7.38 & 5.45 & 7.48 & 7.03 \\
\hline 100 average & & & & 9.68 & 7.50 & 5.43 & 7.55 & 7.34 \\
\hline 100 S.D. & & & & 0.20 & 0.32 & 0.14 & 0.24 & 0.58 \\
\hline 101 & C0011D-13H-7W, 75-77 & 123.151 & 123.162 & 9.97 & 7.66 & 5.41 & 7.70 & 7.60 \\
\hline 102 & C0011D-14H-2W, 89-91 & 126.999 & 127.016 & 9.93 & 7.85 & 5.73 & 7.85 & 7.86 \\
\hline 103 & C0011D-14H-3W, 88-90 & 128.218 & 128.235 & 9.69 & 7.34 & 5.04 & 7.38 & 7.17 \\
\hline 104 & C0011D-14H-4W, 92-94 & 129.481 & 129.498 & 9.78 & 7.34 & 4.86 & 7.35 & 7.50 \\
\hline 105 & C0011D-14H-6W, 88-90 & 130.696 & 130.713 & 9.67 & 7.41 & 5.31 & 7.48 & 7.24 \\
\hline 106 & C0011D-14H-7W, 95-97 & 132.011 & 132.028 & 10.07 & 7.95 & 5.48 & 7.87 & 8.42 \\
\hline 107 & C0011D-14H-8W, 39-41 & 132.751 & 132.768 & 9.48 & 7.18 & 4.94 & 7.22 & 7.21 \\
\hline 108 & C0011D-14H-8W, 55-57 & 132.890 & 132.908 & 8.71 & 5.79 & 3.96 & 6.08 & 5.31 \\
\hline 109 & C0011D-15H-1W, 109-111 & 134.523 & 134.542 & 9.99 & 7.74 & 5.52 & 7.77 & 7.73 \\
\hline 110 & C0011D-15H-2W, 99-101 & 135.762 & 135.781 & 9.81 & 7.50 & 4.95 & 7.46 & 7.70 \\
\hline 110 rep. & & & & 9.83 & 7.45 & 4.88 & 7.42 & 7.67 \\
\hline 110 rep. & & & & 9.74 & 7.45 & 4.98 & 7.43 & 7.49 \\
\hline 110 average & & & & 9.79 & 7.46 & 4.94 & 7.44 & 7.62 \\
\hline 110 S.D. & & & & 0.05 & 0.03 & 0.05 & 0.02 & 0.11 \\
\hline 111 & C0011D-15H-3W, 86-88 & 136.983 & 137.001 & 9.86 & 7.78 & 5.61 & 7.77 & 7.87 \\
\hline 112 & C0011D-15H-5W, 85-87 & 138.536 & 138.555 & 9.91 & 7.73 & 5.65 & 7.77 & 7.65 \\
\hline 113 & C0011D-15H-6W, 103-105 & 140.043 & 140.062 & 9.97 & 7.78 & 5.42 & 7.76 & 8.15 \\
\hline 114 & C0011D-15H-6W, 110-112 & 140.109 & 140.128 & 9.96 & 7.71 & 5.61 & 7.77 & 7.58 \\
\hline 115 & C0011D-15H-7W, 100-102 & 141.352 & 141.371 & 9.46 & 7.15 & 5.11 & 7.24 & 7.09 \\
\hline 116 & C0011D-15H-8W, 85-87 & 142.540 & 142.559 & 9.39 & 7.03 & 5.13 & 7.17 & 6.51 \\
\hline 117 & C0011D-16H-1W, 109-111 & 144.040 & 144.059 & 9.45 & 7.14 & 5.18 & 7.25 & 6.81 \\
\hline 118 & C0011D-16H-3W, 79-81 & 145.255 & 145.275 & 9.99 & 7.72 & 5.35 & 7.72 & 7.88 \\
\hline 119 & C0011D-16H-4W, 43-45 & 145.871 & 145.890 & 9.91 & 7.63 & 5.23 & 7.61 & 7.93 \\
\hline 120 & C0011D-16H-5W, 104-106 & 147.230 & 147.249 & 9.43 & 7.21 & 5.35 & 7.32 & 6.96 \\
\hline 120 rep. & & & & 9.65 & 7.48 & 5.50 & 7.55 & 7.31 \\
\hline 120 rep. & & & & 9.44 & 7.10 & 5.22 & 7.24 & 6.70 \\
\hline 120 average & & & & 9.51 & 7.26 & 5.36 & 7.37 & 6.99 \\
\hline 120 S.D. & & & & 0.13 & 0.19 & 0.14 & 0.16 & 0.31 \\
\hline 121 & C0011D-16H-6W, 44-46 & 148.007 & 148.026 & 9.93 & 7.86 & 5.66 & 7.84 & 8.00 \\
\hline 122 & C0011D-17H-1W, 86-88 & 150.725 & 150.742 & 9.91 & 7.76 & 5.46 & 7.74 & 7.89 \\
\hline 123 & C0011D-17H-2W, 83.5-85.5 & 151.910 & 151.927 & 9.58 & 7.32 & 5.37 & 7.42 & 6.96 \\
\hline 124 & C0011D-17H-3W, 14-16 & 152.525 & 152.542 & 10.23 & 8.32 & 5.98 & 8.23 & 8.55 \\
\hline 125 & C0011D-17H-4W, 20-22 & 153.592 & 153.609 & 9.92 & 7.68 & 5.29 & 7.66 & 7.86 \\
\hline 126 & C0011D-17H-5W, 82.5-84.5 & 154.326 & 154.343 & 9.85 & 7.66 & 5.37 & 7.65 & 7.73 \\
\hline 127 & C0011D-17H-7W, 15-17 & 155.148 & 155.165 & 10.20 & 8.11 & 5.69 & 8.04 & 8.48 \\
\hline 128 & C0011D-17H-8W, 25-27 & 156.253 & 156.270 & 10.03 & 7.78 & 5.20 & 7.71 & 8.40 \\
\hline 129 & C0011D-17H-9W, 13-15 & 157.345 & 157.362 & 9.82 & 7.65 & 5.50 & 7.67 & 7.72 \\
\hline 130 & C0011D-18H-2W, 36-38 & 159.043 & 159.062 & 9.42 & 7.16 & 5.38 & 7.30 & 6.76 \\
\hline 130 rep. & & & & 9.59 & 7.31 & 5.22 & 7.38 & 7.10 \\
\hline 130 rep. & & & & 9.63 & 7.40 & 5.71 & 7.56 & 6.93 \\
\hline 130 average & & & & 9.55 & 7.29 & 5.44 & 7.42 & 6.93 \\
\hline 130 S.D. & & & & 0.11 & 0.12 & 0.25 & 0.13 & 0.17 \\
\hline 131 & C0011D-18H-3W, 36-38 & 160.380 & 160.399 & 9.39 & 7.11 & 5.19 & 7.22 & 6.71 \\
\hline 132 & C0011D-18H-5W, 40-42 & 161.984 & 162.003 & 9.72 & 7.44 & 5.23 & 7.48 & 7.45 \\
\hline 133 & C0011D-18H-6W, 32-34 & 163.255 & 163.274 & 9.98 & 7.83 & 5.46 & 7.79 & 8.14 \\
\hline 134 & C0011D-18H-7W, 32-34 & 164.607 & 164.626 & 9.60 & 7.26 & 5.00 & 7.30 & 7.37 \\
\hline 135 & C0011D-18H-8W, 32-34 & 165.953 & 165.972 & 9.96 & 8.04 & 5.95 & 8.01 & 8.10 \\
\hline 136 & C0011D-19H-1W, 113-115 & 168.892 & 168.908 & 9.92 & 8.02 & 5.87 & 7.98 & 8.12 \\
\hline 137 & C0011D-19H-2W, 100-102 & 169.898 & 169.914 & 9.67 & 7.57 & 5.55 & 7.61 & 7.53 \\
\hline 138 & C0011D-19H-4W, 108-110 & 171.263 & 171.279 & 9.79 & 7.80 & 5.47 & 7.74 & 7.90 \\
\hline 139 & C0011D-19H-5W, 74-76 & 172.123 & 172.139 & 9.94 & 8.04 & 5.92 & 8.00 & 8.11 \\
\hline 140 & C0011D-19H-6W, 80-82 & 173.280 & 173.295 & 9.54 & 7.32 & 5.37 & 7.41 & 7.15 \\
\hline 140 rep. & & & & 9.52 & 7.32 & 5.24 & 7.37 & 7.30 \\
\hline 140 rep. & & & & 9.50 & 7.31 & 5.34 & 7.39 & 7.18 \\
\hline 140 average & & & & 9.52 & 7.31 & 5.32 & 7.39 & 7.21 \\
\hline 140 S.D. & & & & 0.02 & 0.01 & 0.07 & 0.02 & 0.08 \\
\hline 141 & C0011D-20H-2W, 98-100 & 176.296 & 176.314 & 9.61 & 7.43 & 5.34 & 7.47 & 7.54 \\
\hline 142 & C0011D-20H-3W, 85-87 & 177.502 & 177.521 & 9.90 & 7.92 & 5.89 & 7.92 & 7.97 \\
\hline 143 & C0011D-20H-5W, 91-93 & 178.868 & 178.887 & NA & NA & NA & NA & NA \\
\hline 144 & C0011D-20H-6W, 66-68 & 179.971 & 179.990 & 9.77 & 7.63 & 5.48 & 7.64 & 7.57 \\
\hline 145 & C0011D-21H-1W, 109.5-111.5 & 181.289 & 181.304 & 9.89 & 7.85 & 5.96 & 7.90 & 7.75 \\
\hline 146 & C0011D-21H-2W, 66.5-68.5 & 181.988 & 182.003 & 9.72 & 7.74 & 6.00 & 7.81 & 7.59 \\
\hline 147 & C0011D-21H-5W, 32-34 & 183.080 & 183.095 & 9.92 & 7.78 & 5.70 & 7.81 & 7.72 \\
\hline 148 & C0011D-22H-1W, 65.5-67.5 & 184.663 & 184.668 & 9.59 & 7.49 & 5.63 & 7.56 & 7.33 \\
\hline 149 & C0011D-21H-6W, 33-35 & 183.952 & 183.967 & 9.56 & 6.68 & 4.16 & 6.79 & 4.93 \\
\hline
\end{tabular}


Table T1 (continued). (Continued on next page.)

\begin{tabular}{|c|c|c|c|c|c|c|c|c|}
\hline \multirow[b]{2}{*}{ Sample } & \multirow[b]{2}{*}{ Hole, core, section, interval $(\mathrm{cm})$} & \multicolumn{2}{|c|}{ Core depth CSF-B (m) } & \multirow[b]{2}{*}{ 10-percentile } & \multirow[b]{2}{*}{ 50-percentile } & \multirow[b]{2}{*}{ 90-percentile } & \multirow[b]{2}{*}{ Mean } & \multirow[b]{2}{*}{ Mode } \\
\hline & & Top & Bottom & & & & & \\
\hline 150 & C0011D-22H-2W, 20-22 & 184.897 & 184.902 & 8.95 & 6.34 & 4.06 & 6.45 & 6.17 \\
\hline 150 rep. & & & & 9.18 & 6.52 & 4.01 & 6.57 & 6.86 \\
\hline 150 rep. & & & & 8.81 & 6.04 & 3.86 & 6.21 & 5.09 \\
\hline 150 average & & & & 8.98 & 6.30 & 3.98 & 6.41 & 6.04 \\
\hline 150 S.D. & & & & 0.19 & 0.24 & 0.10 & 0.18 & 0.89 \\
\hline 151 & C0011D-23T-1W, 125-127 & 187.250 & 187.270 & 10.07 & 8.08 & 6.01 & 8.07 & 8.17 \\
\hline 152 & C0011D-23T-2W, 99-101 & 188.395 & 188.415 & 9.84 & 7.74 & 5.89 & 7.82 & 7.51 \\
\hline 153 & C0011D-23T-3W, 109-111 & 189.905 & 189.925 & 9.90 & 7.90 & 5.96 & 7.93 & 7.88 \\
\hline 154 & C0011D-24T-1W, 50.5-52.5 & 196.005 & 196.025 & 9.88 & 7.78 & 5.77 & 7.82 & 7.71 \\
\hline 155 & C0011D-24T-2W, 35-37 & 196.765 & 196.785 & 9.84 & 7.71 & 5.64 & 7.74 & 7.63 \\
\hline 156 & C0011D-25T-1W, 69-71 & 200.69 & 200.710 & 9.87 & 7.87 & 6.05 & 7.93 & 7.73 \\
\hline 157 & C0011D-25T-3W, 114-116 & 202.305 & 202.325 & 9.89 & 8.09 & 6.31 & 8.11 & 8.07 \\
\hline 158 & C0011D-25T-4W, 97-99 & 203.540 & 203.560 & 9.94 & 7.84 & 5.70 & 7.85 & 7.87 \\
\hline 159 & C0011D-26X-1W, 109-111 & 205.656 & 205.668 & 10.08 & 8.00 & 5.92 & 8.02 & 8.03 \\
\hline 160 & C0011D-26X-2W, 127-129 & 206.613 & 206.624 & 9.88 & 7.75 & 5.70 & 7.78 & 7.67 \\
\hline 160 rep. & & & & 9.95 & 7.89 & 5.84 & 7.90 & 7.90 \\
\hline 160 rep. & & & & 9.97 & 7.87 & 5.77 & 7.88 & 7.86 \\
\hline 160 average & & & & 9.93 & 7.84 & 5.77 & 7.85 & 7.81 \\
\hline 160 S.D. & & & & 0.05 & 0.08 & 0.07 & 0.07 & 0.12 \\
\hline 161 & C0011D-26X-4W, 68-70 & 207.301 & 207.314 & 9.92 & 7.98 & 6.24 & 8.04 & 7.84 \\
\hline 162 & C0011D-26X-5W, 74-76 & 207.990 & 208.002 & 10.00 & 8.02 & 5.96 & 8.01 & 8.05 \\
\hline 163 & C0011D-27X-1W, 44-46 & 210.330 & 210.345 & 10.01 & 7.93 & 5.94 & 7.96 & 7.88 \\
\hline 164 & C0011D-26X-6W, 109-111 & 209.046 & 209.058 & 9.91 & 7.90 & 5.91 & 7.92 & 7.88 \\
\hline 165 & C0011D-27X-2W, 52-54 & 211.445 & 211.460 & 9.82 & 7.86 & 6.18 & 7.95 & 7.69 \\
\hline 166 & C0011D-26X-7W, 10-12 & 209.296 & 209.308 & 9.97 & 7.90 & 6.01 & 7.95 & 7.71 \\
\hline 167 & C0011D-27X-4W, 17-19 & 212.508 & 212.523 & 9.75 & 7.71 & 5.71 & 7.74 & 7.71 \\
\hline 168 & C0011D-27X-5W, 60-62 & 213.656 & 213.671 & 9.96 & 8.00 & 6.07 & 8.02 & 7.97 \\
\hline 169 & C0011D-28X-1W, 32-35 & 215.296 & 215.324 & 9.99 & 7.96 & 5.88 & 7.96 & 7.96 \\
\hline 170 & C0011D-27X-6W, 40-42 & 214.561 & 214.576 & 9.42 & 6.91 & 4.65 & 7.00 & 6.84 \\
\hline 170 rep. & & & & 9.28 & 6.83 & 4.72 & 6.94 & 6.65 \\
\hline 170 rep. & & & & 9.38 & 7.00 & 4.82 & 7.07 & 6.92 \\
\hline 170 average & & & & 9.36 & 6.91 & 4.73 & 7.00 & 6.80 \\
\hline 170 S.D. & & & & 0.07 & 0.08 & 0.09 & 0.07 & 0.14 \\
\hline 171 & C0011D-28X-2W, 50-52 & 216.773 & 216.792 & 9.38 & 6.87 & 4.94 & 7.04 & 6.02 \\
\hline 172 & C0011D-28X-3W, 67-69 & 218.232 & 218.250 & 9.57 & 7.34 & 5.35 & 7.42 & 7.09 \\
\hline 173 & C0011D-28X-4W, 73-75 & 219.593 & 219.611 & 9.38 & 6.81 & 4.57 & 6.92 & 6.40 \\
\hline 174 & C0011D-29X-1W, 81-83 & 220.690 & 220.707 & 9.48 & 7.17 & 5.19 & 7.28 & 6.84 \\
\hline 175 & C0011D-29X-2W, 82-84 & 221.895 & 221.912 & 9.52 & 7.35 & 5.36 & 7.42 & 7.36 \\
\hline 176 & C0011D-29X-3W, 40-42 & 222.738 & 222.756 & 9.51 & 7.39 & 5.57 & 7.49 & 7.18 \\
\hline 177 & C0011D-29X-5W, 37-39 & 223.923 & 223.939 & 9.49 & 7.18 & 4.88 & 7.20 & 7.42 \\
\hline 178 & C0011D-30X-1W, 48-50 & 225.480 & 225.500 & 9.71 & 7.55 & 5.27 & 7.54 & 7.70 \\
\hline 179 & C0011D-29X-CC, 12-13 & 224.732 & 224.740 & 9.92 & 7.86 & 5.79 & 7.87 & 7.86 \\
\hline 180 & C0011D-30X-2W, 65-67 & 227.050 & 227.070 & 9.90 & 7.73 & 5.71 & 7.78 & 7.60 \\
\hline 180 rep. & & & & 9.84 & 7.70 & 5.83 & 7.79 & 7.42 \\
\hline 180 rep. & & & & 9.97 & 7.79 & 5.78 & 7.85 & 7.67 \\
\hline 180 average & & & & 9.90 & 7.74 & 5.77 & 7.81 & 7.56 \\
\hline 180 S.D. & & & & 0.06 & 0.05 & 0.06 & 0.04 & 0.13 \\
\hline 181 & C0011D-30X-3W, 85-87 & 228.475 & 228.495 & 9.84 & 7.77 & 5.68 & 7.78 & 7.80 \\
\hline 182 & C0011D-31X-1W, 14-16 & 234.640 & 234.660 & 9.65 & 7.47 & 5.52 & 7.55 & 7.37 \\
\hline 183 & C0011D-31X-2W, 14-16 & 236.025 & 236.045 & 9.24 & 6.73 & 4.56 & 6.84 & 6.70 \\
\hline 184 & C0011D-31X-3W, 14-16 & 237.435 & 237.455 & 10.05 & 8.02 & 5.89 & 8.00 & 8.14 \\
\hline 185 & C0011D-31X-5W, 29-31 & 238.740 & 238.760 & 9.99 & 7.59 & 5.54 & 7.70 & 7.42 \\
\hline 186 & C0011D-31X-6W, 14-16 & 240.000 & 240.020 & 10.00 & 8.06 & 5.90 & 8.01 & 8.34 \\
\hline 187 & C0011D-32X-1W, 127-129 & 242.638 & 242.656 & 9.49 & 7.13 & 4.92 & 7.19 & 7.24 \\
\hline 188 & C0011D-32X-2W, 127-129 & 243.897 & 243.915 & 10.02 & 8.03 & 5.99 & 8.02 & 8.11 \\
\hline 189 & C0011D-32X-5W, 134-136 & 245.756 & 245.774 & 10.23 & 8.18 & 6.02 & 8.16 & 8.50 \\
\hline 190 & C0011D-33X-1W, 75-77 & 247.196 & 247.214 & 9.68 & 7.52 & 5.47 & 7.57 & 7.54 \\
\hline 190 rep. & & & & 9.73 & 7.54 & 5.49 & 7.59 & 7.54 \\
\hline 190 rep. & & & & 9.60 & 7.39 & 5.36 & 7.46 & 7.36 \\
\hline 190 average & & & & 9.67 & 7.48 & 5.44 & 7.54 & 7.48 \\
\hline 190 S.D. & & & & 0.06 & 0.08 & 0.07 & 0.07 & 0.10 \\
\hline 191 & C0011D-33X-2W, 50-52 & 248.249 & 248.267 & 10.07 & 8.15 & 6.13 & 8.13 & 8.27 \\
\hline 192 & C0011D-33X-4W, 34-36 & 249.487 & 249.506 & 9.68 & 7.50 & 5.60 & 7.60 & 7.22 \\
\hline 193 & C0011D-33X-5W, 32-34 & 250.767 & 250.786 & 10.16 & 8.15 & 6.08 & 8.14 & 8.29 \\
\hline 194 & C0011D-34X-1W, 72-74 & 251.997 & 252.010 & 9.82 & 7.90 & 6.09 & 7.94 & 7.82 \\
\hline 195 & C0011D-34X-2W, 98-100 & 253.145 & 253.159 & 9.67 & 7.48 & 5.48 & 7.55 & 7.53 \\
\hline 196 & C0011D-34X-3W, 81-83 & 254.000 & 254.014 & 10.28 & 8.25 & 6.09 & 8.22 & 8.51 \\
\hline 197 & C0011D-35X-1W, 42-44 & 256.920 & 256.940 & 10.28 & 8.18 & 5.99 & 8.17 & 8.37 \\
\hline 198 & C0011D-34X-5W, 87-89 & 255.290 & 255.303 & 10.21 & 8.15 & 6.00 & 8.13 & 8.20 \\
\hline
\end{tabular}


Table T1 (continued). (Continued on next page.)

\begin{tabular}{|c|c|c|c|c|c|c|c|c|}
\hline \multirow[b]{2}{*}{ Sample } & \multirow[b]{2}{*}{ Hole, core, section, interval $(\mathrm{cm})$} & \multicolumn{2}{|c|}{ Core depth CSF-B (m) } & \multirow[b]{2}{*}{ 10-percentile } & \multirow[b]{2}{*}{ 50-percentile } & \multirow[b]{2}{*}{ 90-percentile } & \multirow[b]{2}{*}{ Mean } & \multirow[b]{2}{*}{ Mode } \\
\hline & & Top & Bottom & & & & & \\
\hline 199 & C0011D-34X-6W, 73-75 & 256.165 & 256.179 & 10.20 & 8.09 & 5.85 & 8.06 & 8.35 \\
\hline 200 & C0011D-36X-1W, 115.5-117.5 & 267.087 & 267.106 & 9.79 & 7.68 & 5.74 & 7.74 & 7.58 \\
\hline 200 rep. & & & & 9.64 & 7.54 & 5.69 & 7.62 & 7.38 \\
\hline 200 rep. & & & & 9.60 & 7.48 & 5.69 & 7.58 & 7.24 \\
\hline 200 average & & & & 9.68 & 7.57 & 5.71 & 7.64 & 7.40 \\
\hline 200 S.D. & & & & 0.10 & 0.10 & 0.03 & 0.08 & 0.17 \\
\hline 201 & C0011D-36X-2W, 133.5-135.5 & 268.580 & 268.599 & 10.01 & 8.04 & 6.04 & 8.05 & 8.05 \\
\hline 202 & C0011D-36X-3W, 60.5-62.5 & 269.229 & 269.248 & 10.02 & 7.96 & 5.93 & 7.98 & 7.94 \\
\hline 203 & C0011D-36X-4W, 59.5-61.5 & 270.543 & 270.562 & 10.05 & 8.17 & 6.27 & 8.17 & 8.19 \\
\hline 204 & C0011D-36X-6W, 78-80 & 272.045 & 272.063 & 9.77 & 7.77 & 5.74 & 7.78 & 7.79 \\
\hline 205 & C0011D-36X-6W, 90.5-92.5 & 272.162 & 272.181 & 9.94 & 7.91 & 5.83 & 7.91 & 7.98 \\
\hline 206 & C0011D-36X-7W, 103-105 & 273.608 & 273.626 & 10.09 & 8.10 & 6.09 & 8.11 & 8.15 \\
\hline 207 & C0011D-36X-8W, 83-85 & 274.747 & 274.766 & 10.00 & 8.01 & 5.99 & 8.02 & 8.07 \\
\hline 208 & C0011D-37X-1W, 86-88 & 276.288 & 276.306 & 10.01 & 8.04 & 6.05 & 8.05 & 8.09 \\
\hline 209 & C0011D-37X-2W, 85-87 & 277.543 & 277.561 & 10.07 & 8.05 & 6.03 & 8.06 & 8.07 \\
\hline 210 & C0011D-37X-3W, 130-132 & 279.247 & 279.266 & 10.00 & 8.01 & 5.94 & 8.00 & 8.21 \\
\hline 210 rep. & & & & 10.04 & 8.01 & 6.00 & 8.03 & 8.05 \\
\hline 210 rep. & & & & 9.94 & 7.90 & 5.82 & 7.90 & 8.00 \\
\hline 210 average & & & & 9.99 & 7.97 & 5.92 & 7.98 & 8.09 \\
\hline 210 S.D. & & & & 0.05 & 0.06 & 0.09 & 0.06 & 0.11 \\
\hline 211 & C0011D-37X-5W, 71-73 & 280.315 & 280.333 & 9.96 & 8.01 & 6.04 & 8.02 & 8.04 \\
\hline 212 & C0011D-37X-6W, 72-74 & 281.314 & 281.332 & 10.05 & 8.09 & 6.14 & 8.11 & 8.12 \\
\hline 213 & C0011D-37X-7W, 51-53 & 282.042 & 282.060 & 10.13 & 8.17 & 6.12 & 8.16 & 8.37 \\
\hline 214 & C0011D-38X-1W, 113-115 & 283.630 & 283.650 & 9.92 & 8.03 & 6.16 & 8.05 & 8.04 \\
\hline 215 & C0011D-38X-2W, 114-116 & 285.050 & 285.070 & 9.77 & 7.87 & 6.13 & 7.92 & 7.76 \\
\hline 216 & C0011D-38X-4W, 20-22 & 285.890 & 285.910 & 9.96 & 8.09 & 6.20 & 8.09 & 8.09 \\
\hline 217 & C0011D-38X-5W, 134-136 & 287.615 & 287.635 & 9.91 & 7.95 & 5.89 & 7.94 & 8.03 \\
\hline 218 & C0011D-38X-6W, 67-69 & 288.345 & 288.365 & 10.08 & 8.05 & 6.00 & 8.06 & 8.05 \\
\hline 219 & C0011D-38X-7W, 10-12 & 289.255 & 289.275 & 9.88 & 7.99 & 6.21 & 8.03 & 7.90 \\
\hline 220 & C0011D-38X-CC, 7-9 & 289.765 & 289.785 & 10.09 & 8.23 & 6.35 & 8.24 & 8.26 \\
\hline 220 rep. & & & & 10.08 & 8.17 & 6.22 & 8.17 & 8.22 \\
\hline 220 rep. & & & & 9.90 & 7.95 & 6.07 & 7.98 & 7.90 \\
\hline 220 average & & & & 10.02 & 8.12 & 6.21 & 8.13 & 8.13 \\
\hline 220 S.D. & & & & 0.10 & 0.15 & 0.14 & 0.13 & 0.20 \\
\hline 221 & C0011D-39X-1W, 76-78 & 292.729 & 292.748 & 9.91 & 7.99 & 6.17 & 8.03 & 7.90 \\
\hline 222 & C0011D-39X-2W, 86-88 & 294.167 & 294.186 & 10.07 & 8.18 & 6.20 & 8.17 & 8.28 \\
\hline 223 & C0011D-39X-3W, 104-105 & 295.691 & 295.700 & 10.10 & 8.10 & 6.08 & 8.10 & 8.15 \\
\hline 224 & C0011D-39X-4W, 94-96 & 296.937 & 296.956 & 10.04 & 8.20 & 6.30 & 8.20 & 8.24 \\
\hline 225 & C0011D-39X-6W, 77-79 & 298.135 & 298.154 & 9.93 & 8.04 & 6.13 & 8.05 & 8.05 \\
\hline 226 & C0011D-39X-7W, 96-98 & 299.679 & 299.698 & 9.98 & 8.05 & 6.12 & 8.06 & 8.02 \\
\hline 227 & C0011D-39X-8W, 45-47 & 300.546 & 300.565 & 10.01 & 8.05 & 5.96 & 8.03 & 8.18 \\
\hline 228 & C0011D-40X-1W, 111-113 & 302.549 & 302.568 & 9.95 & 8.10 & 6.15 & 8.08 & 8.17 \\
\hline 229 & C0011D-40X-2W, 95-97 & 303.726 & 303.745 & 10.04 & 8.15 & 6.02 & 8.10 & 8.36 \\
\hline 230 & C0011D-40X-3W, 67-69 & 304.799 & 304.818 & 10.06 & 8.08 & 5.81 & 8.02 & 8.36 \\
\hline 230 rep. & & & & 10.02 & 8.05 & 5.86 & 8.00 & 8.23 \\
\hline 230 rep. & & & & 9.95 & 7.98 & 5.87 & 7.96 & 8.08 \\
\hline 230 average & & & & 10.01 & 8.04 & 5.85 & 7.99 & 8.22 \\
\hline 230 S.D. & & & & 0.06 & 0.05 & 0.03 & 0.03 & 0.14 \\
\hline 231 & C0011D-40X-4W, 63-65 & 306.094 & 306.113 & 9.90 & 7.98 & 6.11 & 8.00 & 7.94 \\
\hline 232 & C0011D-40X-6W, 71-73 & 307.328 & 307.346 & 10.02 & 8.20 & 6.36 & 8.20 & 8.21 \\
\hline 233 & C0011D-40X-7W, 67-69 & 308.623 & 308.642 & 10.03 & 8.15 & 6.11 & 8.12 & 8.22 \\
\hline 234 & C0011D-40X-8W, 75-77 & 310.031 & 310.050 & 9.92 & 8.01 & 6.11 & 8.03 & 8.00 \\
\hline 235 & C0011D-41X-1W, 117-119 & 312.170 & 312.190 & 10.04 & 8.13 & 6.15 & 8.12 & 8.16 \\
\hline 236 & C0011D-41X-2W, 127-129 & 313.620 & 313.640 & 9.96 & 8.39 & 6.98 & 8.45 & 8.26 \\
\hline 237 & C0011D-41X-3W, 96-98 & 314.720 & 314.740 & 10.07 & 8.12 & 6.15 & 8.13 & 8.10 \\
\hline 238 & C0011D-41X-4W, 41-43 & 315.585 & 315.605 & 10.16 & 8.20 & 6.01 & 8.16 & 8.38 \\
\hline 239 & C0011D-41X-6W, 74-76 & 317.515 & 317.535 & 9.90 & 8.08 & 6.66 & 8.21 & 7.84 \\
\hline 240 & C0011D-41X-7W, 35-37 & 318.535 & 318.555 & 10.00 & 8.15 & 6.32 & 8.16 & 8.12 \\
\hline 240 rep. & & & & 9.98 & 8.03 & 5.99 & 8.02 & 8.10 \\
\hline 240 rep. & & & & 9.96 & 8.04 & 5.97 & 8.01 & 8.12 \\
\hline 240 average & & & & 9.98 & 8.07 & 6.09 & 8.06 & 8.11 \\
\hline 240 S.D. & & & & 0.02 & 0.07 & 0.19 & 0.09 & 0.01 \\
\hline 241 & C0011D-42X-1W, 46.5-48.5 & 320.443 & 320.462 & 10.02 & 8.05 & 6.03 & 8.06 & 8.08 \\
\hline 242 & C0011D-42X-2W, 53-55 & 321.844 & 321.863 & 10.08 & 8.13 & 5.94 & 8.08 & 8.26 \\
\hline 243 & C0011D-42X-3W, 55-57 & 323.197 & 323.216 & 10.08 & 8.11 & 5.94 & 8.07 & 8.32 \\
\hline 244 & C0011D-42X-5W, 117-119 & 325.517 & 325.536 & 9.98 & 7.91 & 5.86 & 7.93 & 7.88 \\
\hline 245 & C0011D-42X-6W, 12-14 & 325.860 & 325.879 & 10.06 & 8.13 & 6.11 & 8.12 & 8.20 \\
\hline 246 & C0011D-42X-7W, 13-15 & 327.208 & 327.227 & 10.12 & 8.26 & 6.33 & 8.25 & 8.27 \\
\hline 247 & C0011D-42X-8W, 21-23 & 328.623 & 328.642 & 9.96 & 8.06 & 6.17 & 8.08 & 8.03 \\
\hline
\end{tabular}


Table T1 (continued).

\begin{tabular}{|c|c|c|c|c|c|c|c|c|}
\hline \multirow[b]{2}{*}{ Sample } & \multirow[b]{2}{*}{ Hole, core, section, interval (cm) } & \multicolumn{2}{|c|}{ Core depth CSF-B (m) } & \multirow[b]{2}{*}{ 10-percentile } & \multirow[b]{2}{*}{ 50-percentile } & \multirow[b]{2}{*}{ 90-percentile } & \multirow[b]{2}{*}{ Mean } & \multirow[b]{2}{*}{ Mode } \\
\hline & & Top & Bottom & & & & & \\
\hline 248 & C0011D-43X-1W, 14-16 & 329.615 & 329.631 & 9.92 & 8.21 & 6.38 & 8.18 & 8.22 \\
\hline 249 & C0011D-43X-2W, 12-14 & 330.732 & 330.748 & 10.03 & 7.94 & 5.83 & 7.95 & 7.97 \\
\hline 250 & C0011D-43X-4W, 58-60 & 332.579 & 332.595 & 9.71 & 7.78 & 6.13 & 7.86 & 7.57 \\
\hline 250 rep. & & & & 9.79 & 7.81 & 5.94 & 7.85 & 7.71 \\
\hline 250 rep. & & & & 9.78 & 7.81 & 6.02 & 7.87 & 7.66 \\
\hline 250 average & & & & 9.76 & 7.80 & 6.03 & 7.86 & 7.65 \\
\hline 250 S.D. & & & & 0.05 & 0.02 & 0.10 & 0.01 & 0.07 \\
\hline 251 & C0011D-43X-5W, 62-64 & 333.765 & 333.782 & 9.89 & 7.95 & 5.97 & 7.96 & 7.99 \\
\hline 252 & C0011D-44X-1W, 49-51 & 334.912 & 334.929 & 9.79 & 7.71 & 6.09 & 7.84 & 7.27 \\
\hline 253 & C0011D-44X-2W, 36-38 & 335.987 & 336.004 & 9.93 & 7.95 & 5.82 & 7.93 & 8.06 \\
\hline 254 & C0011D-44X-3W, 3-5 & 336.891 & 336.908 & 9.99 & 8.00 & 5.78 & 7.96 & 8.32 \\
\hline 255 & C0011D-44X-5W, 61-63 & 338.055 & 338.072 & 9.96 & 8.00 & 5.85 & 7.97 & 8.15 \\
\hline 256 & C0011D-44X-6W, 45-47 & 338.450 & 338.466 & 9.65 & 7.48 & 5.11 & 7.44 & 7.96 \\
\hline 257 & C0011D-45X-1W, 24-26 & 339.720 & 339.738 & 10.00 & 8.19 & 6.55 & 8.24 & 8.06 \\
\hline 258 & C0011D-44X-6W, 112-114 & 339.013 & 339.029 & 9.96 & 7.97 & 5.78 & 7.93 & 8.09 \\
\hline 259 & C0011D-44X-CC, 4-6 & 339.240 & 339.256 & 9.97 & 8.15 & 6.60 & 8.23 & 7.95 \\
\hline 260 & C0011D-45X-2W, 3-5 & 340.816 & 340.835 & 9.99 & 8.06 & 6.06 & 8.06 & 8.11 \\
\hline 260 rep. & & & & 9.91 & 7.89 & 5.84 & 7.89 & 7.96 \\
\hline 260 rep. & & & & 10.18 & 8.19 & 5.95 & 8.13 & 8.52 \\
\hline 260 average & & & & 10.02 & 8.05 & 5.95 & 8.03 & 8.20 \\
\hline 260 S.D. & & & & 0.14 & 0.15 & 0.11 & 0.12 & 0.29 \\
\hline 261 & C0011D-45X-4W, 5-7 & 342.500 & 342.518 & 10.05 & 8.18 & 6.52 & 8.25 & 8.03 \\
\hline 262 & C0011D-45X-5W, 55-57 & 343.890 & 343.908 & 9.95 & 8.08 & 6.49 & 8.16 & 7.88 \\
\hline 263 & C0011D-45X-CC, 5-7 & 344.147 & 344.165 & 10.05 & 8.00 & 5.76 & 7.96 & 8.25 \\
\hline 264 & C0011D-46X-1W, 33-35 & 344.804 & 344.823 & 9.98 & 8.04 & 5.94 & 8.02 & 8.18 \\
\hline 265 & C0011D-46X-2W, 25-27 & 346.022 & 346.041 & 9.93 & 7.99 & 5.91 & 7.97 & 8.08 \\
\hline 266 & C0011D-46X-4W, 19-21 & 347.277 & 347.295 & 9.92 & 7.99 & 5.90 & 7.96 & 8.11 \\
\hline 267 & C0011D-47X-3W, 18-20 & 351.447 & 351.462 & 8.55 & 5.25 & 3.57 & 5.67 & 4.41 \\
\hline 268 & C0011D-47X-4W, 27-29 & 351.818 & 351.833 & 10.00 & 8.07 & 6.31 & 8.13 & 7.96 \\
\hline 269 & C0011D-47X-6W, 19-21 & 352.489 & 352.504 & 9.95 & 7.04 & 4.87 & 7.24 & 5.46 \\
\hline 270 & C0011D-49X-1W, 56-58 & 359.954 & 359.970 & 10.10 & 8.01 & 5.67 & 7.96 & 8.37 \\
\hline 270 rep. & & & & 10.04 & 8.00 & 5.77 & 7.97 & 8.29 \\
\hline 270 rep. & & & & 9.89 & 7.84 & 5.67 & 7.83 & 7.97 \\
\hline 270 average & & & & 10.01 & 7.95 & 5.70 & 7.92 & 8.21 \\
\hline 270 S.D. & & & & 0.11 & 0.10 & 0.06 & 0.08 & 0.21 \\
\hline 271 & C0011D-49X-2W, 38-40 & 360.936 & 360.952 & 9.97 & 8.00 & 5.86 & 7.97 & 8.14 \\
\hline 272 & C0011D-49X-4W, 14-16 & 362.278 & 362.295 & 9.76 & 7.71 & 6.09 & 7.84 & 7.35 \\
\hline 273 & C0011D-49X-5W, 6-8 & 363.357 & 363.374 & 9.26 & 6.67 & 4.92 & 6.92 & 5.75 \\
\hline 274 & C0011D-52X-2W, 55-57 & 376.945 & 376.965 & 10.03 & 8.02 & 5.86 & 7.99 & 8.21 \\
\hline 275 & C0011D-52X-3W, 41-43 & 378.220 & 378.240 & 10.01 & 8.06 & 5.95 & 8.03 & 8.22 \\
\hline 276 & C0011D-52X-5W, 31-33 & 379.260 & 379.280 & 10.04 & 8.11 & 6.05 & 8.09 & 8.27 \\
\hline 277 & C0011D-52X-CC, 12-14 & 379.700 & 379.720 & 10.02 & 8.18 & 6.30 & 8.18 & 8.21 \\
\hline
\end{tabular}

rep. $=$ repeat measurement, S.D. $=$ standard deviation. $\mathrm{NA}=$ not applicable . 